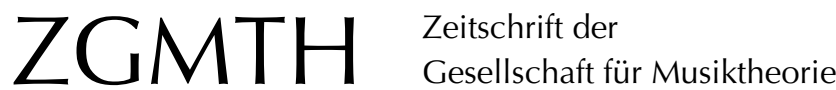

Schwab-Felisch, Oliver (2007): Umriss eines allgemeinen Begriffs des musikalischen Satzmodells. ZGMTH 4/3, 291-304. https://doi.org/10.31751/262

(C) 2007 Oliver Schwab-Felisch

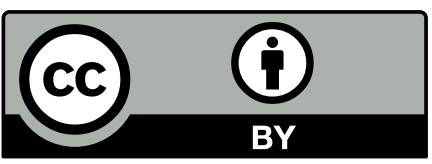

Dieser Text erscheint im Open Access und ist lizenziert unter einer Creative Commons Namensnennung 4.0 International Lizenz.

This is an open access article licensed under a

Creative Commons Attribution 4.0 International License.

veröffentlicht / first published: 01/07/2007

zuletzt geändert / last updated: 09/08/2009 


\title{
Umriss eines allgemeinen Begriffs des musikalischen Satzmodells
}

\author{
Oliver Schwab-Felisch
}

Ausgehend von einem Begriff des musikalischen Modells als sstrukturaler Repräsentation eines individuellen Werkausschnitts wird im ersten Teil des Beitrags schrittweise ein allgemeiner Begriff des musikalischen Satzmodells entwickelt. Der zweite Teil untersucht Aspekte der Instantiierung musikalischer Satzmodelle. Er führt die Begriffe der ıInstanzklasse`, der `Elaboration` und der 'Transformation ein, unterscheidet drei spragmatische Instanztypen Arten, in denen Satzmodelle sich auf die Kontexte 'Form` und 'Struktur beziehen können.

Der Terminus smusikalisches Satzmodelk scheint unproblematisch: Wer ihn kennt, kann Beispiele für Satzmodelle anführen und weiß einzelne musikalische Bildungen als Satzmodelle zu identifizieren. Was Satzmodelle ausmacht, ist freilich weniger klar, als es scheinen mag. Zwar darf als sekundär gelten, dass der Terminus in mehreren Grundbedeutungen auftritt ${ }^{1}$ und auch in seiner Hauptbedeutung "Klangfortschreitungs-Modell $\aleph^{2}$ von Autor zu Autor unterschiedlich akzentuiert wird. Klärungsbedarf besteht aber insofern, als smusikalisches Satzmodelk einer Gruppe von Termini zugehört, die sich einerseits überschneiden, andererseits unterschiedlichen historischen Zusammenhängen entstammen, unterschiedliche Aspekte akzentuieren, unterschiedliche Begriffskontexte implizieren und unterschiedliche Extensionen besitzen. ${ }^{3}$ Erste Resultate der begriffsgeschichtlichen Untersuchungen, nach denen die unübersichtliche Situation verlangt, liegen inzwischen vor. ${ }^{4}$ Was allerdings jene Gebilde unterschiedlichster Herkunft und Beschaffenheit, die als Satzmodelle gelten können, überhaupt als solche qualifiziert, ist

1 Vgl. etwa Roch 2000: „Es gehört aber zu den Widersprüchen der Geschichte, daß sich das jeweils Neue immer nur in den Begriffen des Alten zu definieren vermag. So ist es zu erklären, daß Tieck und andere Frühromantiker ausgerechnet auf das der Affektenlehre entgegengesetzte musikalische Satzmodell der alten Vokalpolyphonie zurückgreifen, um von daher die neuere Instrumentalmusik zu legitimieren«. - Die Polysemie des Ausdrucks ımusikalisches Satzmodelk erinnert an die des unspezifischeren Ausdrucks >musikalisches Modellı, der u. a. in den Bedeutungen 'Sinnbild bestehender oder wünschenswerter außermusikalischer Sachverhalte`, ssinguläres Vorbild einer singulären Komposition`, sstilbildendes Werkı, ‘Variationsthema‘, sbeispielhafter Stilı, 'Set von Standardverfahren zur Lösung bestimmter kompositorischer Aufgaben male einer Mehrzahl von Kompositionen` verwendet wird.

2 Fladt 2005, 189.

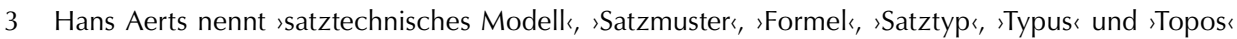
(2007). Historische und fremdsprachliche Termini wären zu ergänzen.

4 Vgl. Aerts 2007. 
bislang erst in Ansätzen diskutiert worden. Zu fragen wäre etwa nach der Bestimmung des Begriffspaars 'Modellı und ıInstanzı, nach dem Verhältnis der Begriffe >Modellı, 'Klasse`, 'Schema füge strukturbildender Elemente, den pragmatischen Funktionen von Modellinstanzen oder dem Einfluss kompositorischer Kontexte auf ihre Gestalt. Hier setzt der vorliegende Text an. Er skizziert Grundbestimmungen des musikalischen Modellbegriffs, macht einen Vorschlag zur Bestimmung des systematischen Ortes von Satzmodellen und beleuchtet einige elementare Eigenschaften von Modellinstanzen.

\section{>Abbild}

\section{Strukturerhaltende Abbildung}

Ob Architektur oder Kognitionswissenschaft, Informatik oder mathematische Modelltheorie: viele Disziplinen verfügen über Modellbegriffe, und es fehlt nicht an Versuchen, sie zu systematisieren. ${ }^{5}$ So unterscheidet Herbert Stachowiaks Allgemeine Modelltheorie ${ }^{6}$, die wohl immer noch wichtigste Publikation zum Thema, drei grundlegende Merkmale von Modellen:

- „Abbildungsmerkmal«: »Modelle sind stets Modelle von etwas, nämlich Abbildungen, Repräsentationen natürlicher oder künstlicher Originale, die selbst wieder Modelle sein können. ${ }^{7}$

_ „Verkürzungsmerkmal«: »Modelle erfassen im allgemeinen nicht alle Attribute des durch sie repräsentierten Originals, sondern nur solche, die den jeweiligen Modellerschaffern und/oder Modellbenutzern relevant erscheinen. $\aleph^{8}$

5 Siehe Müller 2001. - Modellbegriffe anderer Disziplinen sind von eminentem Interesse auch für die Musiktheorie. Von ihnen her, nicht aus dem Begriffsfundus des Fachs erschließen sich die mathematischen, modelltheoretischen, klassenlogischen, symboltheoretischen, kognitionspsychologischen und ontologischen Aspekte, die das Verhältnis zwischen Modell und Gegenstand umfasst. Zwar betreffen sie die Geschichte und Verwendungsweise einzelner Modelle nur indirekt. Aber sie können erhellen, was es heißt, in analytischem Kontext mit Modellen umzugehen.

6 Stachowiak 1973.

7 Ebd., 131. - Der Begriff der Abbildung ist verschiedentlich kritisiert worden, so etwa bei Hans Jörg Sandkühler: »Fragwürdig ist, dass viele Philosophen Bilder als Abbilder verstehen, gerade so, als stünden die erkennenden Subjekte außerhalb einer fertigen Welt, die sie nur noch sabzubilden hätten. Um den Sachverhalt in einem Bild auszudrücken: Wann immer wir uns Bilder von der Welt machen, sind wir im Bild. Wir kopieren nicht, sondern wir entwerfen, und unsere Bilder sind immer auch Selbstbilder und Selbstentwürfe« (2003, 48). Allerdings betont auch Stachowiak den Konstruktcharakter des Originals: "Modell in jenem mehrfach zu relativierenden Sinne ist ebenso die selementarste`Wahrnehmungs`gegebenheit‘ wie die komplizierteste, umfassendste Theorie. Modell ist das vermeintlich objektive Erkenntnisgebilde ebenso wie die Gedankenkonstruktion, die ihre Subjektivität und Perspektivität betont« $(1973,56)$. Andreas Bartels verweist auf die ontologische Indifferenz des Begriffs der strukturalen Repräsentation (2005, 189-196). Da es im gegebenen Zusammenhang allein um die formale Relation zwischen dem Modell und seinem Bezugsgegenstand geht, steht der ontologische Aspekt nicht zur Diskussion.

8 Stachowiak 1973, 132. 
- »Pragmatisches Merkma/«: »Modelle sind ihren Originalen nicht per se eindeutig zugeordnet. Sie erfüllen ihre Ersetzungsfunktion a) für bestimmte - erkennende und/oder handelnde, modellbenutzende - Subjekte, b) innerhalb bestimmter Zeitintervalle und c) unter Einschränkung auf bestimmte gedankliche oder tatsächliche Operationen. ${ }^{9}$

Das analytische Diagramm eines singulären Werkausschnitts weist alle drei Modellmerkmale auf: Es ist eine sstrukturerhaltende Abbildung، des originalen Werkausschnitts, enthält weniger Elemente und Relationen als dieser und dient dazu, auf jene Eigenschaften des Originals zu verweisen, die es exemplifiziert (und damit zugleich auch auf jene, die es nicht exemplifiziert). ${ }^{10}$

\section{Idealisierung}

Ein analytisches Diagramm, das die strukturtragenden Töne des originalen Werkausschnitts exakt darstellte, wäre von einer Fassung des originalen Werkausschnitts, in der alle nichtstrukturellen Töne gelöscht sind, nicht zu unterscheiden. Freilich ließe sich das exakte Modell eines auch nur durchschnittlich stark diminuierten Originals lediglich mit Mühe entziffern: Es zeigte eine von Pausen durchsetzte, scheinbar willkürlich über das Notenblatt geworfene Ansammlung von Einzelereignissen. Modelle werden daher häufig snormalisiert ${ }^{11}$ oder sidealisiert.$^{12}$ Zur Idealisierung freigegeben sind stets die Eigenschaften des Modells, die keine konstitutiven Eigenschaften der repräsentierten Struktur sind. Die absoluten Tonhöhen etwa eines durch Intervallklassen ${ }^{13}$ und metrische Gewichte bestimmten Tonhöhenmodells sind variabel, solange die Intervallklassen gewahrt bleiben, die zeitlichen Bestimmungen seiner Klänge sind es, solange sie den Unterschied metrisch betonter und unbetonter Zählzeiten erkennen lassen.

9 Ebd., $132 \mathrm{f}$.

10 Soll die Repräsentationsbeziehung als solche untersucht werden, ist es unerheblich, welche struktualen Eigenschaften aus welchen Gründen repräsentiert werden (vgl. Bartels 2005, 54 f.) - In seiner von Nelson Goodman beeinflussten Bildtheorie weist Oliver Scholz die These zurück, das Isomorphieprinzip könne als allgemeines Prinzip piktorialer Repräsentation verstanden werden (2004, 74-78). Bartels dagegen hebt hervor, eine "strukturerhaltende Abbildung« sei nicht als Isomorphismus, sondern als Homomorphismus aufzufassen: „Homomorphismen machen Urbild und Bild homomorph zueinander, aber Homomorphie ist keine Ähnlichkeit (d.h. sie ist keine symmetrische Relation) « $(2005,24)$. Die Diskussion muss in unserem Zusammenhang nicht weiter interessieren, weil hier eine spezifische Relation beschrieben und kein Anspruch auf Allgemeingültigkeit erhoben wird, das Konzept also nicht jeden möglichen Fall abdecken muss.

11 Rothstein 1990.

12 ıIdealisierung`verändert Werte wie etwa die Dauern im Interesse einer vereinfachten Darstellung. >Abstraktion ersetzt Werte durch Variablen; dadurch werden sie aus einer konkreten Beschreibung gelöscht (vgl. Bartels 2005, 88).

13 Unter sIntervallklasse soll hier die Klasse eines Intervalls und seiner Oktaverweiterungen verstanden werden. 


\section{Generalisierung}

Da das Strukturmodell als einmal Konstituiertes nicht mehr von dem originalen Werkausschnitt abhängt, lässt es sich auf alle Werkausschnitte beziehen, deren Strukturen dieselben Eigenschaften besitzen wie es selbst. ${ }^{14}$ Wird Übereinstimmung sowohl hinsichtlich der Struktur als auch der konkreten Eigenschaften ihrer Instanz gefordert, ist der Kreis der denotierten Werkausschnitte eng begrenzt. Gilt hingegen allein die strukturelle Übereinstimmung als Kriterium, erweitert er sich erheblich.

Der Bezug auf mehrere Gegenstände ${ }^{15}$ verändert das Modell, ohne seine materiale Beschaffenheit zu beeinflussen. Da die denotierten Werkausschnitte einander allein in struktureller Hinsicht gleichen, können die nichtstrukturellen Elemente des Modells nicht mehr auf nichtstrukturelle Elemente der Werkausschnitte abgebildet werden. Tonhöhe, Dauer oder metrische Position eines Modelltons werden zu variablen Größen, die konkrete Gestalt des Modells erscheint als gleichsam zufälliges Exempel einer Vielzahl divergenter Einzelfälle, die Struktur löst sich von ihrem materiellen Substrat. >Modelk heißt nun auch das Abstraktum, welches das singuläre konkrete Modell verkörpert. Alle Modelle, die sich auf mehrere musikalische Einheiten beziehen, sind in diesem Sinne abstrakt.

Das Abstraktum >Modell lässt sich auf dreierlei Weise auffassen, und zwar als

- Element der Klasse aller musikalischen Gebilde, welche die Charakteristika eines musikalischen Modells aufweisen,

- kognitives Schemar, also hierarchisch organisierte kognitive Struktur mit der Funktion, Repräsentationen der Merkmale eines Gegenstandes, Sachverhaltes oder Vorgangs kognitiv verfügbar zu machen ${ }^{16}$ und

- eine bestimmte Art eines abstrakten Gegenstandes. ${ }^{17}$

14 Welche Gegenstände das Strukturmodell denotiert, bestimmt der Verwendungszusammenhang.

15 Stimmen mehrere Modelle hinsichtlich ihrer Struktur miteinander überein und stimmt jedes dieser Modelle hinsichtlich seiner Struktur mit seinem Bezugsgegenstand überein, muss auch jedes dieser Modelle hinsichtlich seiner Struktur mit jedem der Bezugsgegenstände der anderen Modelle übereinstimmen.

16 Siehe etwa Neisser 1979; Brewer/Nakamura 1984; Rumelhart 1984; Gjerdingen 1988; Leman 1995; Lenk 1998; Smith/Queller 2001. - Der Ausdruck `Schema ‘ wird zur Bezeichnung sowohl kognitiver als auch musikalischer Strukturen verwendet. In musiktheoretischen Zusammenhängen scheint es sinnvoll, sich auf die Bedeutung skognitives Schemar zu beschränken.

17 >Abstrakte Gegenstände` wie »Beethovens Opus 90, das Fahrrad, die österreichische Fahne und die Sachertorte« haben laut Maria E. Reicher ein »Merkmal gemeinsam: Sie alle sind vielfach instantiierbar." Reicher weiter: »Die Instantiierungen von Beethovens Opus 90 sind konkrete musikalische Aufführungen, die Instantiierungen des Fahrrades sind konkrete Fahrräder, die Instantiierungen der Sachertorte sind konkrete Sachertorten, und so fort. Gegenstände, die vielfach instantiierbar sind, nenne ich Typen. Beethovens Opus 90 ist also ein Typus, der in musikalischen Aufführungen instantiiert sein kann, dessen Existenz aber nicht von der Existenz etwaiger Instantiierungen abhängt. (Beethovens Opus 90 existiert auch dann, wenn es gerade nirgendwo auf der Welt aufgeführt wird). Jede korrekte Aufführung von Beethovens Opus 90 ist eine Instantiierung von Beethovens Opus 90. Typen zeichnen sich dadurch aus, dass sie determinierende Eigenschaften haben« (Reicher 2005, 252 f.) - Eigenschaften, die im Fall eines musikalischen Typus' »determinieren, welche 
Die ontologischen Implikationen der Termini `Klasse`, 'Schema und `Typus` werden im Folgenden ausgeklammert, die Termini pragmatisch gebraucht. Von `Klasse sprochen, wenn extensionale Aspekte im Vordergrund stehen, etwa wenn eine Menge von Gegenständen in mehrere Mengen geringeren Umfangs zu differenzieren ist, von 'Schemaı, wo die Repräsentation eines Modells im kognitiven Apparat einer Person angesprochen wird und von `Typus`, wenn die Beziehung zwischen dem Abstraktum sModell und seinen Instanzen als Gegenstandsbeziehung modelliert werden soll. Keiner dieser Termini soll den eingeführten Terminus >Modell ersetzen.

In den vorangegangenen Abschnitten waren wir zunächst von dem Strukturmodell eines singulären Werkausschnitts ausgegangen und hatten seinen Gegenstandsbereich in einem zweiten Schritt auf die Klasse aller strukturidentischen Werkausschnitte erweitert. In einem dritten Schritt wäre nun nach den Kriterien zu fragen, die es erlauben, die Gesamtheit aller Satzmodelle unter einen Begriff zu subsumieren und sie zugleich von der Gesamtheit aller anderen Modelle zu unterscheiden. Diese Kriterien sind mit den gemeinsamen Merkmalen der musikalischen Gebilde, die entsprechend einem vorgängigen Begriff von 'Satzmodellı als Satzmodelle gelten können, nicht gleichzusetzen: Jede Instanz einer Quintfallsequenz besitzt eine spezifische Ausdehnung; gleichwohl würde niemand behaupten wollen, Ausdehnung sei ein Unterscheidungsmerkmal von Quintfallsequenzen. Zwar führt das datengeleitete Verfahren zur Bildung einer exakt definierten Klasse, doch steht nicht fest, ob diese Klasse mit derjenigen der Satzmodelle identisch ist. Der Begriff des Satzmodells soll daher im Folgenden durch schrittweise Spezifikation bestimmt werden. ${ }^{18}$

\section{Zur Bestimmung des Typus smusikalisches Satzmodell}

\section{Musikalisches Modell}

Die Klasse smusikalisches Modell wird durch eine zweifache Spezifikation aus der Klasse aller Kompositionen abgeleitet. Die erste Spezifikation erzeugt die Klasse aller Strukturen, die diesen Kompositionen überhaupt zugesprochen werden können. Dabei ist offen, wie viele Elemente an der Konstitution einer Struktur beteiligt sind und zu wie vielen Strukturen ein musikalisches Element gehört. Die zweite Spezifikation greift aus der Klasse der Strukturen die Klasse der musikalischen Modelle heraus. Ein musikalisches Modell ist die Klasse aller kompositorischen Einzelfälle oder Instanzen, die einander strukturell gleichen. Die Klasse ‘musikalisches Modelk enthält die Gesamtheit aller musikalischen Modelle.

Eigenschaften jede korrekte Aufführung des betreffenden Werks erfüllen muss«. (Ebd., 251) - Siehe auch Tugendhat 1976, 161-175, 497-521; Stegmüller 1978.

18 Es wird im Folgenden jeweils nur die Klasse einer Klassifikationsebene skizziert, die im Hinblick auf die Fragestellung von Interesse ist. - Die naheliegende Frage, ob ein allgemeiner Begriff des Satzmodells möglich ist oder die einzelnen Modellbegriffe vielmehr nach dem Wittgensteinschen Prinzip der `Familienähnlichkeit` zusammenhängen, klärt sich über Erfolg oder Scheitern des Versuchs, einen allgemeinen Begriff des Satzmodells zu bestimmen. 
Tonhöhe

Über die Unterscheidung einfacher und komplexer musikalischer Parameter wie `Tonhöhe`, `Tondauerı, `Textur modelle Intervallrelationen determinieren, greifen wir im Folgenden die Klasse der Tonhöhenmodelle heraus.

\section{Exkurs: Formmodell versus Satzmodell}

Konstituenten musikalischer Formen werden im Allgemeinen über die Zuordnung genereller, bisweilen durch einen Index erweiterter Termini wie etwa ^Motiv a bezeichnet. Für sich genommen ist die Bezeichnung >Motiv as unspezifisch. Was sie in einem bestimmten Kontext bedeutet, wissen wir erst, wenn wir die Tonfolge kennen, die sie bezeichnet. Wir ordnen eine uninterpretierte Tongruppe, indem wir die Kategorie des Motivs auf sie anwenden, erhalten einzelne Motiv-Individuen und bezeichnen sie gemäß ihrer Zugehörigkeit zu einer Motivklasse. Erst die Verbindung von Notentext und generellem Terminus ergibt den Namen des Motivs. Der generelle Aspekt eines Motivs wird mithin verbal denotiert, der singuläre Aspekt nonverbal exemplifiziert. Nur in besonderen Fällen erhalten Motive Eigennamen.

Die Bezeichnung musikalischer Konstituenten erfolgt nach unterschiedlichen Gesichtspunkten. Termini wie `Periode` oder `Sonatensatzform ‘ fokussieren strukturelle, Termini wie `Überleitung` oder `Schlussgruppe` funktionale Aspekte. Funktional bestimmte Elemente des Sonatensatzschemas können durch Bezeichnung ihres strukturellen Aspekts spezifiziert werden - so wie in dem Satz »Das erste Thema instantiiert den Typus der Periode».

Die Intension des Terminus `Periode` wird ebenso durch Strukturmerkmale bestimmt wie die des Terminus `Quintfallsequenzı. Und doch unterscheiden sich Periode und Quintfallsequenz grundsätzlich. Der Typus >Periode` determiniert unter anderem Anzahlen, Relationen und Wirkungen - Eigenschaften, die Töne voraussetzen, auf deren Konstellationen sie sich manifestieren können. Die determinierenden Eigenschaften der Quintfallsequenz dagegen sind Anordnungen von Intervallen. Sie setzen keine festgelegten Tonfolgen voraus, sondern erzeugen sie erst: Wer der Aufforderung folgen möchte, die Unterquinte des Tons c zu bilden, muss einen Ton hervorbringen, dessen Tonhöhe eine Quinte unter c liegt. Satzmodelle bestimmen die Generation konkreter Tonhöhen, indem sie konkrete Intervallrelationen vorgeben. Dies gilt auch dort, wo abstrakte Akkorde mit im Spiel sind.${ }^{19}$ Alle Intervalle und Intervallkonfigurationen, die ein Satzmodell determiniert, sind konkret instantiierbar. Ihre Abfolge ist so bestimmt, dass die Abfolge der Simultaneinheiten einer seinfachen Instanzı einen lückenlosen Zusammenhang darstellt. ${ }^{20}$

19 Abstrakte Akkorde sind Klassen konkreter Akkorde. Jeder konkrete Akkord kann als Produkt der Anwendung einer Transpositions-, Lösch- und Vervielfältigungsfunktion auf die durch die Akkordbezeichnung gegebene Grundmenge von Tönen aufgefasst werden. Die Grundmenge bestimmt das Was, die Funktionen das Wo und das Wieviel.

20 Eine seinfache Instanz` erfüllt die determinierenden Eigenschaften ihres Typus und wählt für alle anderen Eigenschaften die jeweils einfachsten Werte. Siehe Abschnitt `Pragmatische Bestimmung von Instanzen<. 


\section{Materialordnung}

Eine Unterteilung der Klasse der Tonhöhenmodelle nach dem Kriterium der Materialordnung entspricht insofern der Sachlage, als sämtliche unter dem Begriff des Satzmodells diskutierten Gebilde das Regelsystem des Kontrapunkts unmittelbar oder mittelbar voraussetzen und mit dem Ende der Tonalität zumindest prinzipiell außer Gebrauch geraten. Innerhalb des Zeitraums von ca. 1450 bis heute weitere Zäsuren zu setzen, scheint in unserem Zusammenhang nicht sinnvoll. ${ }^{21}$ Zwar verlieren Modelle und Instanzklassen ${ }^{22}$ an Aktualität, treten bislang unbekannte hinzu. Die größere Zahl von Satzmodellen aber besteht über musikhistorische Zäsuren hinweg. Änderungen der Musiksprache manifestieren sich nicht in ihrer Substanz, sondern der Art ihrer Instantiierung und Kontextualisierung. ${ }^{23}$ Das charakteristisch Ungesättigte der Modelle erlaubt synchronisch wie diachronisch unzählige Realisationsformen. Sie alle sind zu spezifisch, um in die Bestimmung eines allgemeinen Begriffs des Satzmodells eingehen zu können.

Intertextualität

Immanente Äquivalenzrelationen, wie sie zwischen einem Motiv, einer Phrase oder einem Formteil und ihren Wiederholungen bestehen, vermögen ungeachtet der Tatsache, dass dem jeweiligen ıUrbild Modellcharakter im umgangssprachlichen Sinne zukommt, kein Satzmodell zu begründen. Satzmodelle setzen Äquivalenzrelationen zwischen Strukturen verschiedener abgeschlossener musikalischer Einheiten voraus.

\section{Allgemeinheit}

Allerdings führen nicht alle intertextuellen Äquivalenzrelationen zur Ausbildung eines Satzmodells. Eine kompositorische Bezugnahme auf individuelle Eigenschaften einer musikalischen Einheit erzeugt keine Instanz eines Satzmodells, sondern eine Variation, ein Zitat oder eine Entlehnung. ${ }^{24}$ Satzmodelle aber sind allgemein. Ihre Allgemeinheit hat einen quantitativen und einen qualitativen Aspekt. Ersterer verlangt, dass modellkonstitutive Gemeinsamkeiten zwischen einer hinreichend großen Anzahl von Elementen bestehen, letzterer, dass sie nicht durch spezifische Details, sondern bestimmte Grundzüge gebildet werden. Beide Aspekte gelten nicht absolut, sondern wirken zusammen: Auch tausend Zitate einer individuellen Passage machen diese noch nicht zu einem Satzmodell, ein einziges Zitat einer individuellen diminuierbaren Struktur dagegen legt

21 Vgl. Sachs 1984, 231-256.

22 Siehe unten, Abschnitt ıInstanzklasse`.

23 Vgl. Froebe 2007.

24 Eine analytische Bezugnahme auf individuelle Eigenschaften einer musikalischen Einheit erzeugt womöglich ein Modell im Sinne der allgemeinen Modelltheorie. Ein analytisches Modell einer musikalischen Einheit ist aber nur dann eine Instanz eines Satzmodells, wenn diese eine Instanz eines Satzmodells ist und das analytische Modell das Satzmodell modelliert. - Dass Zitate individueller Wendungen keine Satzmodelle sind, heißt nicht, dass Satzmodelle nicht zitiert werden können. 
gleichsam den Grundstein zu einem Satzmodell, das als solches bestätigt wird, wenn hinreichend viele Kompositionen von ihm Gebrauch machen.

\section{Diminuierbarkeit}

Da lokale musikalische Gebilde in vielerlei Hinsicht andere Eigenschaften besitzen als globale, bietet sich `Ausdehnung، als weiterer Klassifikator an. Angesichts von Phänomenen wie der innerhalb eines einzigen Taktes durchschrittenen Quintfallsequenz ${ }^{25}$ darf allerdings bezweifelt werden, ob sich eine untere Grenze der Ausdehnung hinreichend klar bestimmen ließe. ${ }^{26}$ Sinnvoller scheint es, den Klassifikator `Ausdehnung، aufzuspalten und die Entscheidung, ob es sich bei Einheiten von geringer Ausdehnung um Satzmodelle handelt, an das Kriterium der Diminuierbarkeit zu binden. Als `diminuierbar sollen Typen gelten, die sowohl in einfachen als auch in diminuierten komplexen Instanzen, als snicht diminuierbar Typen, die nur in einfachen Instanzen vorkommen: weitgehend exakt definierte melodische Folgen, die gleichsam nur zitiert, aber nicht paraphrasiert werden. Diminutionsfiguren wie Triller und Doppelschläge, aber auch Joachim Brügges »Typen A, B und $\mathrm{C}^{27}$ sind Beispiele für nicht diminuierbare Tonhöhenmodelle, Gegenbeispiele bieten der Schenkersche Ursatz, eine abstrakte Harmoniefolge oder eine beliebige > Ligaturenketter.

\section{Mittlere Ausdehnung}

Tonhöhenmodelle lassen sich hinsichtlich ihrer Ausdehnung in globale und lokale unterteilen. Ein Modell ist global, wenn es unter Berücksichtigung aller Elemente einer Komposition gebildet wurde, lokal, wenn ihm lediglich ein Ausschnitt zugrundeliegt. Zwischen der Ausdehnung eines Modells und seiner Allgemeinheit besteht ein gewisser Zusammenhang. Globale Tonhöhenmodelle sind nur ausnahmsweise generell - so etwa der Schenkersche Ursatz. Eine vollständige Analyse nach Heinrich Schenker dagegen, ein Baumdiagramm nach Lerdahl und Jackendoff oder eine Melodieanalyse nach Eugene Narmour sind ebenso singulär wie das Werk, auf das sie sich beziehen. Satzmodelle sind lokale generelle Tonhöhenmodelle.

\section{Mehrstimmigkeit}

In der Musik seit ca. 1450 fungieren einstimmige Strukturen zumeist als Komponenten mehrstimmiger Sätze oder Darstellungen mehrstimmiger Strukturen ${ }^{28}$, sind also von mehrstimmigen Strukturen abhängig. Strukturen genuin einstimmiger Musik bilden einen

25 Johann Sebastian Bach, Das wohltemperierte Klavier Teil II, Fuge E-Dur, T. 39.

26 Unklar ist bereits, wie die Ausdehnung eines Satzmodells zu bestimmen ist: anhand der Anzahl aufeinanderfolgender Klänge oder besetzter Zählzeiten, anhand der Relation zur Gesamtlänge einer Komposition, zur Länge eines ihrer Abschnitte, zu seiner eigenen Ereignisdichte oder shierarchischen Tiefes?

27 Brügge 1993, 185.

28 Siehe Schwab-Felisch 2007. 
eigenen Strukturtyp und damit eine eigene Klasse. Harmoniemodelle sind Verbindungen abstrakter Harmonien. Sie ebenfalls separat zu klassifizieren, läge nahe. Allerdings sind abstrakte Harmonieverbindungen Klassen konkreter Stimmführungskonstellationen: Es gibt keine Instanz einer abstrakten Harmonieverbindung ohne konkrete Stimmführung. Stimmführung aber ist nicht willkürlich: die Vielfalt des satztechnisch Zulässigen kann durch Remineszenzen an die Stimmführung eines zugrundeliegenden Kontrapunktmodells, Beachtung des simaginären Continuo ${ }^{29}$ oder neu kristallisierte Stimmführungsmodelle reguliert werden. Die Angabe einer abstrakten Stufenfolge ist mithin grundsätzlich unvollständig: eine pragmatisch motivierte Abstraktion der Analyse.

Damit kommen wir zu einer Definition: Ein Satzmodell ist ein musikalischer Typus, der als intertextuelle, generelle, diminuierbare und mehrstimmige Intervallstruktur eines Ausschnitts einer nach ca. 1450 entstandenen modalen oder harmonisch tonalen Komposition bestimmt ist. ${ }^{30}$

Unterschiedliche Arten von Satzmodellen normativ zu klassifizieren, erscheint nicht sinnvoll. Selbstverständlich ließen sich mit dem Klassifikator 'Satztechnikı Bassmodelle und Intervallsatzmodelle, mit dem Klassifikator `Tonvorrat ‘ diatonische und chromatische und mit dem Klassifikator Iteration sequenzielle und nicht-sequenzielle Modelle differenzieren. Doch zeitigten diese Unterscheidungen keine stabilen Resultate: Jedes Satzmodell fällt in mehrere Klassen, und es hängt von der Fragestellung ab, welche Klasse jeweils aktualisiert wird.

\section{`Vorbild}

\section{Elaboration}

Bisher haben wir >Modell im Wesentlichen als ein Zweites, als idealisierte `Abbildung der Struktur eines musikalischen Abschnitts oder Produkt der Zusammenfassung musikalischer Einheiten mit gleicher Struktur behandelt. Damit ist freilich nur eine von zwei Grundrelationen zwischen dem Modell und seinem Bezugsgegenstand erfasst. Bereits im Begriff des Typus ist ja mitgedacht, dass der abstrakte Gegenstand nicht allein über einen nachträglichen Akt der Abstraktion zustandekommt, sondern zugleich auch der Hervorbringung von Instanzen zugrundeliegt. >Modelk ist demnach ebenso ein Erstes wie ein Zweites, `Vorbild

Im Allgemeinen erfüllt eine Modellinstanz die determinierenden Eigenschaften ihres Typus und ergänzt die Eigenschaften, ohne die ein konkretes Exemplar des Typus nicht existieren könnte. `Einfache Instanzen beschränken sich auf genau diese Funktion: Sie wählen für alle Eigenschaften, über die sie selbst entscheiden, die jeweils einfachsten Bestimmungen. ${ }^{32}$ `Elaborierte` Instanzen dagegen können aus einer größeren Anzahl von

29 Vgl. Rothstein 1991, Cadwallader/Gagné 2007, 62 f.

30 Vgl. die Aufstellungen bei Fladt 2005, Kaiser 2007a, Gjerdingen 2007, Froebe 2007.

31 Vgl. Stachowiak 1973, 129; Bartels 2005, 10.

32 'Keine Vorzeichen`, seinfacher Kontrapunktı, 'gerader Taktı, smittleres Registerı, senge Lage` etc. 
Tönen bestehen, als es gemäß der determinierenden Eigenschaften ihres Typus erforderlich ist, sowie andere Oktavlagen und metrische Positionen wählen. ${ }^{33}$

,Einfache Instanzen` begegnen in drei Spielarten und Funktionen:

- Im Kontext Theorie exemplifizieren sie ihren Typus. In dieser Funktion entscheiden sie über alle Eigenschaften, die keine determinierenden Eigenschaften des Typus sind.

- Im Kontext `Analyse schnitts. In dieser Funktion entscheiden sie über genau die Eigenschaften, durch die sich eine Idealisierung von einer exakten Kopie der strukturtragenden Töne des Originals unterscheidet.

- Im Kontext «Komposition schließlich fungieren sie als Bestandteil einer bestimmten Komposition. In dieser Funktion entscheiden sie allein über die Eigenschaften, die nicht schon durch den kompositorischen Kontext bestimmt sind.

Mit der Unterscheidung der drei Spielarten einfacher Instanzen sind funktionale Kriterien deutlich geworden, nach denen Instanzen generell klassifiziert werden können. Zur Bezeichnung einer Instanz, die ihren Typus exemplifiziert, wird daher der Terminus sinstruktive Instanzı, zur Bezeichnung eines Werkausschnitts der Terminus ästhetische Instanzı und zur Bezeichnung eines analytischen Diagramms einer ästhetischen Instanz der Terminus sanalytische Instanz` vorgeschlagen.

\section{Instanzklasse}

Die Unterscheidung zwischen Modell und Instanz gründet in der Differenz struktureller und nicht-struktureller musikalischer Eigenschaften: Jede Instanz besitzt Eigenschaften, die nicht zu den determinierenden Eigenschaften ihres Typus' gehören. Manche Instanzen ähneln sich hinsichtlich dieser Eigenschaften. Ähnliche Instanzen aber lassen sich klassifizieren. >Instanzklassen ihrer Struktur gleich und hinsichtlich ihrer Elaborationsform ähnlich sind. Sie differenzieren die Menge der heterogenen Gebilde, auf die eine Modellbezeichnung zutrifft, nach historischen und/oder systematischen Kriterien. Instanzklassen können Instanzklassen enthalten. Ihre Zahl ist virtuell unendlich, ihre Bildung setzt spezifische Forschungsfragen voraus.

Instanzklassen werden in der musiktheoretischen Literatur für gewöhnlich ebenso als Modelle bezeichnet wie die abstrakteren Strukturen, die ihnen zugrundeliegen. Da Instanzklassen Modelle von geringerem Abstraktionsgrad sind, ist dies nicht falsch, verwischt aber den kategorialen Unterschied, der zwischen Modellen und Instanzklassen besteht: Während Modelle ausschließlich über ihre Intervallstruktur bestimmt sind, haben Instanzklassen zusätzlich beliebige weitere Momente als determinierende Eigenschaften.

33 Elaboration ist häufig, aber nicht immer gleichbedeutend mit Dimimution: Die ersten fünf Harmonien des Seitenthemas der Waldsteinsonate etwa elaborieren den Typus >Dur-Moll-Parallelismus` durch texturale Verdichtung und metrische Verschiebung, nicht aber durch Interpolation zusätzlicher Töne. 


\section{Transformation}

Ein musikalisches Gebilde, das hinreichend viele, aber nicht sämtliche determinierenden Eigenschaften eines Satzmodells und/oder einer Instanzklasse ausprägt, ist eine Instanz eines stransformierten Satzmodells und/oder einer stransformierten Instanzklasse. Transformationen können durch Erweiterungen, Kürzungen oder Elisionen, Vertauschungen, Verschiebungen oder Lagenänderungen hervorgerufen werden. Sie sind Mittel der kompositorischen Individuation und Movens stilistischer Änderungen zugleich: Die Transformation von Instanzklassen kann zur Bildung neuer Instanzklassen führen, die Transformation von Satzmodellen zur Bildung neuer Satzmodelle.

\section{Kategoriale Bestimmung individueller musikalischer Gebilde}

Eine umfassende Bestimmung der Instanz eines Satzmodells beinhaltet bei Berücksichtigung aller bisher getroffenen Unterscheidungen die Angabe

- des Modellnamens ( Modell $x \iota)$

- der Instanzklasse (>Instanzklasse $y<)$

- des Transformationsstatus (১regulär//transformiert`)

- des Elaborationsstatus (`einfach`/>komplexı)

- des pragmatischen Instanztyps (`instruktiv`//ästhetisch`/ sanalytisch`)

Ein Großteil dieser Bestimmungen muss im analytischen Kontext nicht eigens explizit gemacht werden. Um der logischen Klarheit willen scheint es aber angebracht, die kategorialen Unterschiede von `Typusı, ıInstanzklasse

\section{Modell und Kontext}

Zwei der Kontexte, innerhalb derer Modellinstanzen erscheinen, seien im Folgenden herausgegriffen: `Form` im Sinne der Formtheorie und `Struktur im Sinne der Schichtenlehre Heinrich Schenkers.

Die Einbindung einer Instanz in einen kompositorischen Zusammenhang erfolgt in aller Regel ohne substanzielle Änderungen. Eine `Minimalkadenzı etwa kann im Kontext `Form ‘ als Anfangswendung ${ }^{34}$, im Kontext `Struktur als Prolongation des Tonikadreiklangs fungieren. Allerdings sind Instanzen von Satzmodellen keine neutralen Bausteine. Außenstimmensatz, Diatonik, kadenzielle Bestimmungen und andere Strukturqualitäten können bereits bestimmte Kontexte implizieren. ${ }^{35}$ Eine besondere Situation ergibt sich dort, wo die Kontextualisierung den Kontextimplikationen eines Satzmodells widerspricht oder mehrere Instanzen eines Satzmodells durch verschiedene Kontexte verschiedene Bedeutungen zugewiesen bekommen. Ein Beispiel gibt Ulrich Kaiser in dieser Ausgabe der ZGMTH: eine Instanz eines Modells »pendelt« in einem Kontext um die Tonika he-

34 Siehe Caplin 1998, $24 \mathrm{f}$.

35 Instanzen von Satzmodellen besitzen Kontextimplikation teils von sich aus, teils, weil sich ihr Kontext bereits auf die Instantiierung ausgewirkt hat. 
rum, eine andere führt in einem anderen Kontext zur Tonart der Oberquinte. ${ }^{36}$ Mit Kaiser ließe sich jede Modellinstanz ix in Kontext $y$ < als Instanz eines eigenen Modells definieren; unter Verwendung der hier vorgeschlagenen Terminologie wäre dagegen von einem Modell und den Instanzen zweier Instanzklassen zu sprechen.

Schließlich kann der Kontext eine Instanz derart verändern, dass es zur Transformation des ihr zugrundeliegenden Typus' kommt. Im II. Satz der Klaviersonate E-Dur D 157 von Franz Schubert etwa wird jedes Sequenzglied des `Dur-Moll-Parallelismus durch eine vorangestellte Vorwegnahme des Gliedes in Quintlage ergänzt, während die Sequenzglieder selbst regulär in Terzlage beginnen. ${ }^{37}$ Es lässt sich zeigen, dass diese doppelte Erweiterung durch Erfordernisse der Form begründet ist. ${ }^{38}$ Dass die Schenkersche tonale Struktur Modellinstanzen zu beeinflussen vermag, liegt auf der Hand: Als diminuierbares Tonhöhenmodell macht sie potentiell von denselben Tönen Gebrauch wie diese. So lässt sich die Höherlegung der Mittelstimme in den ersten Takten des Menuetts aus Haydns Sinfonie Nr. 26 in d-Moll als Ergebnis des Einflusses der Urlinie auf die zugrundeliegende 5-6-Progression interpretieren. ${ }^{39}$

\section{Zum Schluss}

Zum einen suchen Begriffsexplikationen den Intuitionen zu entsprechen, die dem alltäglichen Gebrauch des explizierten Begriffs zugrundeliegen. Zum anderen beinhalten sie auch ein konstruktives Moment: Sie führen eine Ordnung ein, die aus dem explizierten Begriff nicht automatisch hervorgeht und zu einigen seiner Bestimmungen und Verwendungsweisen in Widerspruch steht. Diese Ordnung ist pragmatisch aufzufassen: Ihre Funktion entspricht der eines höherstufigen kognitiven Schemas, das der Strukturierung eines Gegenstandsbereichs dient, gegebenenfalls aber auch modifiziert werden kann.

\section{Literatur}

Aerts, Hans (2007), »>Modell und `Topos` in der deutschsprachigen Musiktheorie seit Riemann«, ZGMTH 4/1-2, 143-158.

Bartels, Andreas (2005), Strukturale Repräsentation, Paderborn: mentis.

Brewer, William F. / Glenn V. Nakamura (1984), »The Nature and Functions of Schemas«, in: Handbook of Social Cognition, hg. von Robert S. Wyer jr. u. Thomas K. Srull, Bd. 1, Hillsdale, NJ: Lawrence Erlbaum, 119-160.

Brügge, Joachim (1993), »Typus und Modell in den Klaviersonaten Wolfgang Amadeus Mozarts«, in: Mozart Studien 3, 143-189.

36 Kaiser 2007b.

37 Takte 9-12.

38 Siehe Schwab-Felisch i. V.

39 Siehe ebd. 
Cadwallader, Allen / David Gagné (2007), Analysis of Tonal Music. A Schenkerian Approach, 2. Aufl., New York/Oxford: Oxford University Press.

Eybl, Martin (1995), Ideologie und Methode. Zum ideengeschichtlichen Kontext von Schenkers Musiktheorie (= Wiener Veröffentlichungen zur Musikwissenschaft 32), Tutzing: Schneider.

Fladt, Hartmut (2005), »Satztechnische Topoi«, ZGMTH 2/2-3, 189-196.

Froebe, Folker (2007), „Satzmodelle des `Contrapunto alla mente und ihre Bedeutung für den Stilwandel um 1600«, ZGMTH 4/1-2, 13-56.

Gjerdingen, Robert (2007), Music in the Galant Style [...], Oxford/New York: Oxford University Press.

(1988), A Classic Turn of Phrase: Music and the Psychology of Convention, Philadelphia: University of Pennsylvania Press.

Goodman, Nelson (1997), Sprachen der Kunst. Entwurf einer Symboltheorie, Frankfurt: Suhrkamp.

— / Catherine Z. Elgin (1993), Revisionen, Frankfurt a. M.: Suhrkamp.

Gülke, Peter (1983), »Introduktion als Widerspruch im System. Zur Dialektik von Thema und Prozessualität bei Beethoven « [1970], in: Ludwig van Beethoven, hg. von Ludwig Finscher, Darmstadt: Wissenschaftliche Buchgesellschaft, 338-387.

Kaiser, Ulrich (2007a), Die Notenbücher der Mozarts als Grundlage der Analyse von W. A. Mozarts Kompositionen 1761-1767, Kassel u. a.: Bärenreiter.

— (2007b), »Was ist ein musikalisches Modell?«, ZGMTH 4/3, 275-290.

Kühne, Thomas (2005), „What is a Model?«, Dagstuhl Seminar Proceedings 04104. http://drops.dagstuhl.de/opus/volltexte/2005/23

Leman, Marc (1995), Music and Schema Theory. Cognitive Foundations of Systematic Musicology (= Springer Series in Information Sciences 31), Berlin u.a.: Springer.

Lenk, Hans (1998), Einführung in die Erkenntnistheorie, München: Fink.

Müller, Roland (2001), »Eine Chronik von Modellgebrauch und Modellbegriff«. http://www.muellerscience.com/NavStart.htm

Plath, Wolfgang (1975), »Typus und Modell«, Mozart-Jahrbuch 1973/74, 145-158.

Reicher, Maria Elisabeth (2005), Referenz, Quantifikation und ontologische Festlegung, Heusenstamm: Ontos.

Roch, Eckhard (2000), »Symphonie und Drama. Ansätze zu einer Kritik des Dramatischen in der Symphonik des 19. Jahrhunderts", Archiv für Musikwissenschaft 57/4, 318-339.

Rothstein, William (1990), »Rhythmic Displacement and Rhythmic Normalization« in: Trends in Schenkerian Research, hg. von Allen Cadwallader, New York: Schirmer, 87-114. (1991), »On Implied Tones«, Music Analysis 10/3, 289-328. 
Rumelhart, David E. (1984), „Schemata and the Cognitive System«, in: Handbook of Social Cognition, hg. von Robert S. Wyer jr. u. Thomas K. Srull, Bd. 1, Hillsdale, NJ: Lawrence Erlbaum, 161-188.

Sachs, Klaus-Jürgen (1984), „Die Contrapunctus-Lehre im 14. und 15. Jahrhundert«, in: Die mittelalterliche Lehre von der Mehrstimmigkeit (= Geschichte der Musiktheorie 5), hg. von Hans Heinrich Eggebrecht u.a., Darmstadt: Wissenschaftliche Buchgesellschaft, 161-256.

Sandkühler, Hans Jörg (2003), »Repräsentation. Die Fragwürdigkeit unserer Bilder von der Welt der Dinge«, in: Repräsentation, Krise der Repräsentation, Paradigmenwechsel. Ein Forschungsprogramm in Philosophie und Wissenschaften, hg. von Silja Freudenberger u. Hans Jörg Sandkühler, Frankfurt a.M. u. a.: Peter Lang 2003, 47-70.

Scholz, Oliver (2004), Bild, Darstellung, Zeichen. Philosophische Theorien bildlicher Darstellung, 2. Aufl., Frankfurt a. M.: Klostermann.

Schwab-Felisch, Oliver (i. V.), „Satzmodelle im Kontext», in: Bericht über den 6. Kongress der GMTH in Weimar, 6.-8. Oktober 2006, hg. von Klaus Heiwolt, Hildesheim u. a.: Olms.

(2007), „Das `Vorspiegeln mehrer Stimmen durch Eine`: symboltheoretische Aspekte virtueller Mehrstimmigkeit", in: Vom Erkennen des Erkannten, Musikalische Analyse und Editionsphilologie. Festschrift für Christian Martin Schmidt, hg. von Friederike Wißmann, Thomas Ahrend u. Heinz von Loesch, Wiesbaden u. a.: Breitkopf \& Härtel, 33-42.

Smith, Eliot R. / Sarah Queller (2001), „Mental Representations", in: Blackwell Handbook of Social Psychology: Intraindividual Processes, Malden, MA u.a.; Blackwell, 111-133.

Stachowiak, Herbert (1973), Allgemeine Modelltheorie, Wien/New York: Springer.

Stegmüller, Wolfgang (Hg.) (1978), Das Universalien-Problem, Darmstadt: Wissenschaftliche Buchgesellschaft.

Stoffer, Thomas H. (1993), "Strukturmodelle», in: Musikpsychologie. Ein Handbuch, hg. von Herbert Bruhn, Rolf Oerter u. Helmut Rösing, Reinbek: Rowohlt 1993, 466-478.

Tugendhat, Ernst / Ursula Wolf (1983), Logisch-semantische Propädeutik, Stuttgart: Philipp Reclam jun.

Tugendhat, Ernst (1976), Vorlesungen zur Einführung in die sprachanalytische Philosophie, Frankfurt: Suhrkamp. 